\title{
A crossover comparison of extended release felodipine with prolonged action nifedipine in hypertension
}

Ileana Moncica, Paul I Oh, Izhar ul Qamar, Dennis Scolnik, Gerald S Arbus, Diane Hebert, J Williamson Balfe, Gideon Koren

\begin{abstract}
In a crossover design, control of blood pressure by extended release felodipine was compared with control by prolonged action nifedipine in 21 children with renal hypertension. Compliance with once daily felodipine was higher than with nifedipine, at $95.6($ SEM 2.7) \% v 78.9 (6.0)\% $(p=0.02)$. Mean diastolic blood pressure was lower during the day with felodipine than with nifedipine, at $77.6(2.4) v 84.4(2.8) \mathrm{mm} \mathrm{Hg}$ $(p=0.05)$. Similarly, blood pressure load (the percentage of the day during which the child had blood pressure exceeding the upper limits of normal for age) was lower for felodipine than for nifedipine: $43.5(5.5) \% v 61.3(6.3) \%$. There was an opposite trend during the night, though this did not reach statistical significance. These data suggest that once a day felodipine is effective in children with hypertension. This may be because of improved compliance.

(Arch Dis Child 1995; 73: 154-156)
\end{abstract}

Keywords: hypertension, felodipine, nifedipine.

Hypertension occurs in 1-3\% of the paediatric population. ${ }^{1}$ In distinction to adults, most of the children under the age of 12 years have a secondary form of hypertension, most commonly due to renal parenchymal or renovascular disease, coarctation of the aorta, or endocrine abnormalities. ${ }^{1}$ The more severe forms of hypertension are seen in children with renal disease either before or after renal transplantation.

Development of antihypertensive medications in paediatric patients has lagged behind treatment in adults. Because of concerns about safety and efficacy, trials of new agents have almost universally excluded children. Traditionally treatment therefore has been limited to $\beta$ blockers and diuretics. ${ }^{2}$ Calcium channel blockers have been widely and effectively used in adults but have only recently been introduced to paediatric patients. The dihydropyridine calcium channel blockers are particularly potent smooth muscle relaxants and arterial vasodilators, and may therefore be especially useful in patients with hypertension. Nifedipine prolonged action (PA), which is given two or three times a day, often in combination with other drugs, has played an important part in the treatment of such patients. ${ }^{34}$ A new dihydropyridine calcium antagonist, felodipine, was introduced in 1991 and has also been found to be effective in adults. ${ }^{56}$ This drug is available as an extended release (ER) formulation which has the advantage of once daily administration and potentially improved compliance.

The issue of compliance with chronic medications in children is a serious problem, and attempts to decrease the frequency of drug dosage are a potential way of improving compliance. ${ }^{7}$ The objective of our study was to evaluate the effectiveness of felodipine-ER (Plendil, Astra Pharma) by comparing it with nifedipine-PA (Adalat, Miles) in the treatment of children with hypertension predominantly due to renal disease, in a randomised crossover design. We also assessed improvement in patients' compliance during use of once daily felodipine-ER. To the best of our knowledge this is the first paediatric study with this agent.

\section{Methods}

PATIENTS

Twenty one hypertensive children between the ages of 6 and 18 years who had been receiving nifedipine-PA treatment were asked to participate in the study. Most of these patients $(18 / 21)$ had undergone renal transplantation. Before initiating this protocol, home and clinic blood pressure had been followed closely and exceeded the 95th centile for normal subjects of matched age and gender for at least four out of seven days of the week with two measurements per day. Patients were excluded if their control or treatment of hypertension had been unstable during the preceding month or if they were unable to swallow the $9 \mathrm{~mm}$ diameter felodipine tablet.

The study protocol was approved by our hospital's research ethics committee.

\section{PROCEDURES}

Eligible patients were randomised by a random number table to receive treatment with felodipine-ER or nifedipine-PA for one month and then crossed over to the other agent. Patients and parents were not blinded to the study drugs because of the different appearance and dosage schedules, but the physicians in the clinic were unaware of the assigned drugs for the duration of the study. The starting dose of felodipine was set at one quarter of the nifedipine daily dose $^{56}$ up to a maximum of $20 \mathrm{mg}$ per day. Dose adjustments were 
Table 1 Comparison of blood pressure control: nifedipine twice daily $\mathrm{v}$ felodipine once daily. Values are means (SEM)

\begin{tabular}{|c|c|c|c|c|}
\hline Compared value & $\begin{array}{l}\text { Nifedipine } \\
\text { therapy } \\
\text { (SEM) }\end{array}$ & $\begin{array}{l}\text { Felodipine } \\
\text { therapy } \\
\text { (SEM) }\end{array}$ & Differences & $\begin{array}{l}\text { Significance } \\
(p)\end{array}$ \\
\hline $\begin{array}{l}\text { ABP - systolic day }(\mathrm{mm} \mathrm{Hg}) \\
\text { ABP - diastolic day }(\mathrm{mm} \mathrm{Hg}) \\
\text { ABP - systolic night }(\mathrm{mm} \mathrm{Hg}) \\
\text { ABP - diastolic night }(\mathrm{mm} \mathrm{Hg}) \\
\% \text { High systolic day } \\
\% \text { High diastolic day } \\
\% \text { High systolic night } \\
\% \text { High diastolic night } \\
\text { Home - systolic day }(\mathrm{mm} \mathrm{Hg}) \\
\text { Home - diastolic day }(\mathrm{mm} \mathrm{Hg})\end{array}$ & $\begin{array}{l}134(3 \cdot 6) \\
84 \cdot 4(2 \cdot 8) \\
123(3 \cdot 9) \\
75 \cdot 5(3) \\
58 \cdot 2(6 \cdot 4) \\
61 \cdot 3(6 \cdot 3) \\
31 \cdot 3(7) \\
44 \cdot 9(8 \cdot 9) \\
125 \cdot 8(2 \cdot 5) \\
77 \cdot 8(1 \cdot 6)\end{array}$ & $\begin{array}{r}128 \cdot 5(3 \cdot 6) \\
77 \cdot 6(2 \cdot 4) \\
125 \cdot 1(3 \cdot 4) \\
81 \cdot 1(2 \cdot 7) \\
49 \cdot 7(6 \cdot 5) \\
43 \cdot 5(5 \cdot 5) \\
44 \cdot 3(8 \cdot 2) \\
53 \cdot 5(8 \cdot 3) \\
123(2 \cdot 8) \\
76 \cdot 2(1 \cdot 9)\end{array}$ & $\begin{array}{l}18(5 \cdot 9) \\
13(7 \cdot 5) \\
2 \cdot 8(2 \cdot 6) \\
1 \cdot 6(1 \cdot 7)\end{array}$ & $\begin{array}{l}\text { NS } \\
0 \cdot 05 \\
\text { NS } \\
\text { NS } \\
\text { NS } \\
0.03 \\
0.04 \\
\text { NS } \\
0.05 \\
0.02\end{array}$ \\
\hline
\end{tabular}

$\mathrm{ABP}=$ ambulatory blood pressure measured continuously for 24 hours; Home=blood pressure measured manually at home by parents $/$ patients; $\%$ High = percentage of time with blood pressure at values greater than mean $+2 \mathrm{SD}$ for age.
pren

made individually depending on blood pressure response and monitoring of adverse effects by an independent physician.

\section{BLOOD PRESSURE MEASUREMENTS}

During the study, patients or parents measured blood pressure in the arm at home three times per day, using an automated device, and recorded the values in a diary. The cuff size was two thirds of the arm length, and all measurements were performed with the child in the sitting position. Blood pressure at each clinic visit was measured in triplicate manually with a mercury sphygmomanometer and with an automated Dinamap recorder (Critikon). Ambulatory blood pressure (ABP) was recorded over a 24 hour period using a Takeda blood pressure monitor model TM 2420 (A and D Engineering) at the end of each month of drug treatment. ABP was analysed separately for daytime ( 8 am to $10 \mathrm{pm}$ ) and night time (10 $\mathrm{pm}$ to $8 \mathrm{am})$.

\section{COMPLIANCE}

Compliance was assessed by the Medication Event Monitoring System (MEMS, Aprex), a computerised tracking device in which an electronic chip in the lid of the container records the date and time that the bottle is opened. The parents and children were not aware of the electronic monitoring device.

\section{OTHER TESTS}

We recorded concomitant antihypertensive drugs such as $\beta$ blockers and diuretics as well as other ongoing medications such as prednisone, cyclosporin, and azathioprine. We also followed changes in body weight and plasma creatinine through the individual courses of therapy.

Table 2 Compliance with the trial medications as measured by the medication event monitoring system. Values are means (SEM)

\begin{tabular}{lllll}
\hline & Nifedipine & Felodipine & Differences & Significance \\
\hline Overall compliance (\%) & $78.9(6)$ & $95 \cdot 6(2 \cdot 7)$ & $16.9(4)$ & 0.02 \\
Day compliance (\%) & $73.5(6 \cdot 6)$ & $95.6(2 \cdot 7)$ & $22 \cdot 0(4 \cdot 2)$ & 0.01 \\
\hline
\end{tabular}

STATISTICAL CONSIDERATIONS AND ANALYSES The primary endpoint of the study was a paired comparison of the mean daytime average ambulatory blood pressure at steady state with felodipine-ER versus nifedipine-PA. We anticipated that the daytime diastolic blood pressure might be lower in the felodipine phase due to a smoother and longer duration of action compared with nifedipine-PA. A sample size of 20 patients was needed for a median effect size (half a SD or around $8 \mathrm{~mm}$ $\mathrm{Hg}$ ) difference in blood pressure between the two drugs to be significant with $\alpha$ of 0.05 and power of $80 \%$. Secondary endpoints were nocturnal blood pressure control assessed on home and ABP, proportions of blood pressure readings on $\mathrm{ABP}$ above the 95 th percentile for age, and assessments of compliance. The paired $t$ test was used for comparisons between the two arms of the study. Data are presented as mean (SEM).

\section{Results}

Both drugs were well tolerated by all participants and there were no dropouts. Children received a mean nifedipine dose of $58.9(5 \cdot 1)$ $\mathrm{mg} / \mathrm{d}$ and felodipine at $13.4(1 \cdot 1) \mathrm{mg} / \mathrm{d}$. There were no differences in the doses of concomitant drugs (other antihypertensives, prednisone, cyclosporin) given during the two arms of the study, and serum creatinine did not differ between the two phases.

During the day, diastolic blood pressure with felodipine was significantly lower than with nifedipine (table 1). A similar trend with systolic blood pressure was not statistically significant. During the night the trends reversed, with nifedipine tending to be associated with lower blood pressure; however, the trend did not reach statistical significance. Similar effects were documented with the percentage of time the blood pressure was above the $95 \%$ percentile for age (table 1).

Compliance (that is, percentage of drug taken $v$ prescribed) was significantly higher with felodipine (table 2). Compliance with the evening dose of nifedipine tended to be better than with the morning dose, at $84 \cdot 7(7 \cdot 7) \% v$ $73 \cdot 5(6 \cdot 6) \%, p=0 \cdot 17$.

\section{Discussion}

This study documents good one month effectiveness of the extended release calcium channel blocker felodipine in children with renal hypertension. The better blood pressure control with felodipine during the day can be explained by the substantially better measured compliance with this once daily drug as compared with the twice daily nifedipine. There is evidence to show that compliance is inversely related to the number of pills taken by patients. ${ }^{7}$ The trend towards better compliance with nifedipine at night than during the day may be explained by more intensive parental supervision in the evening. We have recently documented a similar trend with 6-mercaptopurine in children with leukaemia 
(Lau R, Greenberg M, Koren G. Personal communication).

During the night it is possible that the second dose of nifedipine allowed better control of blood pressure, although the differences were not clinically significant. In our study the felodipine dose was set at one quarter of the nifedipine dose; however, based on our cumulative experience it appears that some children need higher doses to assure optimal control of blood pressure. Recent clinical experience in both children and adults has confirmed these observations.

In summary, these data suggest that once daily felodipine can be successfully given to children with hypertension. It is likely that over longer study periods the differences in compliance in favour of this drug would have been even larger.

GK is a career scientist of The Ontario Ministry of Health. Supported by Astra Pharma Inc, Toronto, Ontario, and MRC, Canada.

1 Dillon MJ. Investigation and management of hypertension in children. Pediatr Nephrol 1987; 1: 59-68.

2 Report of the second task force on blood pressure control in children. Pediatrics 1987; 79: 1-25.

3 Frishman WH, Charlap S. Nifedipine in the treatment of systemic hypertension. Arch Intern Med 1984; 144: 2335-6.

4 Spivak C, Ocken S, Frishman WH. Calcium antagonists: clinical use in the treatment of systemic hypertension. clinical use in the treatme

5 Morgan TO. A review of the antihypertensive effects of felodipine alone or in combination. F Cardiovasc Pharmaco 1990. 15 (suppl 4): S76-84.

6 Todd PA, Faulas D. Felodipine - a review of the pharmacology and therapeutic use of the extended release formulation in cardiovascular disorders. Drugs 1992; 44: 251-77. 7 Thatcher SJ. Medication compliance. Pediatr Clin North Am 1981; 28: 5-21. 